\title{
The imaging assessment and specific endograft design for the endovascular repair of ascending aortic dissection
}

This article was published in the following Dove Press journal:

Clinical Interventions in Aging

14 July 2016

Number of times this article has been viewed

\author{
Yepeng Zhang' \\ Hanfei Tang' \\ JianPing Zhou ${ }^{2}$ \\ Zhao Liu' \\ Changjian Liu' \\ Tong Qiao' \\ Min Zhou'
}

'Department of Vascular Surgery, The Affiliated Hospital of Nanjing University Medical School, Nanjing, ${ }^{2}$ Department of General Surgery, Yixin People's Hospital, Yixin, People's Republic of China
Correspondence: Min Zhou

Department of Vascular Surgery, The Affiliated Hospital of Nanjing University Medical School, Zhongshan Road No 32I, Nanjing 210008, People's Republic of China

Tel +86 I3951937586

Email zhouminnjuseu@sina.com
Background: Endovascular option has been proposed for a very limited and selected number of Stanford type A aortic dissection (TAAD) patients. We have performed a computed tomography (CT)-based TAAD study to explore appropriate endograft configurations for the ascending aortic pathology.

Methods: TAAD patients treated with optimal CT scans were retrospectively reviewed, and their entry tears (ETs) were identified using three-dimensional and multiplanar reconstructions in an EndoSize workstation. After generating a centerline of flow, measurements, including numerous morphologic characteristics of anatomy, were evaluated and a selected subset of patients were determined to be suitable for endovascular treatments. Proximal diameter and distal diameter of endograft were selected based on diameters measured at the ET level and at the innominate artery (IA) level, with $10 \%$ oversizing with respect to the true lumen, but not exceeding the original aortic diameter. The length of the endograft was determined by the distance from the sinotubular junction to IA.

Results: This study covered 126 TAAD patients with primary ET in ascending aorta, among which, according to the assumed criteria, 48 (38.1\%) patients were deemed to be suitable for endovascular treatment. The diameters of ascending aorta from the sinotubular junction to the IA level presented a downward trend, and the proximal diameters differed significantly from distal diameters of the endograft for TAAD (39.9 versus $36.2 \mathrm{~mm}, P<0.01$ ), implying that the conical endograft might be compatible with the ascending pathology. In the ascending aorta, lengths of the endograft should be 50,60, 70, 80, and $90 \mathrm{~mm}$ in five (10.4\%), 22 (45.9\%), 13 (27.1\%), six (12.5\%), and two (4.2\%) patients, respectively.

Conclusion: In this selected number of Chinese patients, the suitability of endovascular repair has been demonstrated based on the CT imaging. Shorter, larger, and bare spring-free conical endografts were preferred in the ascending aortic pathology.

Keywords: type A dissection, endovascular, endograft, design

\section{Introduction}

Stanford type A aortic dissection (TAAD) is a catastrophic clinical condition, occurring mainly in middle and old age groups, which requires emergency open surgical intervention. Although surgical results have improved with improvements in cardiac surgery techniques over the last two decades, overall in-hospital mortality remains as high as $30 \%{ }^{1}$ The older people usually have poor physical condition, and they are often associated with other multiple basic diseases. Therefore, owing to the significant mortality and morbidity of open surgery, patients of high risks are not considered for this procedure. ${ }^{2}$ Previous reports have described that, in a small selected subset 
of patients, stent graft placement in the ascending aorta for TAAD is feasible and may represent a novel effective and safe procedure..$^{3-7}$ Computed tomography (CT) scan imaging studies in European and American population showed that endovascular repair was anatomically feasible in $\sim 30 \%$ of TAAD patients with a proximal entry tear (ET) in the ascending aorta. This percentage of patients may increase up to $39 \%$ with the use of extra-anatomic bypass, such as a left-to-right carotid-carotid bypass, in order to obtain an adequate distal landing zone. ${ }^{8,9}$ Recently, our previous anatomical study suggested that $38 \%$ of Chinese patients with TAAD could potentially be treated by stent grafting. ${ }^{10}$

Current endovascular treatment for TAAD, as with type B aortic dissection, involves sealing the proximal ET and remodeling the dissected aorta but can be more challenging because of complex features of this aortic segment. ${ }^{11-14}$ Endografts should be precisely deployed between the sinotubular junction (STJ) and the innominate artery (IA). However, $\sim 36 \%$ of TAAD patients were not suitable for endovascular treatment due to the absence of available endografts, and endografts specifically designed for ascending aorta are not currently available. ${ }^{9}$ Off-license techniques such as using endografts designed for the descending aorta or aortic cuff are unlikely to offer a robust solution but to compromise outcome. ${ }^{3}$ Traditional thoracic endograft had to be shortened intraoperatively in order not to cover the supraaortic trunks. ${ }^{13}$ The ideal endograft configurations of TAAD should be determined and developed before endovascular treatment can be implemented in the daily practice.

The objective of this study is to perform a CT-based characterization of TAAD with a primary ET in ascending aorta and make a preliminary exploration to the endograft configurations specific for ascending aorta. The delivery system and mechanism of deployment compatible with ascending aortic pathology were also described.

\section{Methods}

This is a retrospective study of CT scan data of all patients treated for TAAD at Nanjing Drum Tower Hospital between 2008 and 2014. Penetrating aortic ulcer (PAU) affecting the ascending aorta was considered a classic dissection, and CT scans of PAU were also included in the study. The exclusion criterion was the absence of a workable (maximum slice thickness of $1.25 \mathrm{~mm}$ and high-quality arterial phase) preoperative CT scan with Digital Imaging and Communications in Medicine (DICOM) data and patients with retrograde TAAD who had primary ET distal to the IA. CT angiography was performed in patients with high-resolution CT operating at $120 \mathrm{kv} / 250 \mathrm{~mA}$ with a $512 \times 512$ matrix. The CT scanning called for injection of $100 \mathrm{~mL}$ of iodinated contrast agent automatically injected with $4 \mathrm{~mL} / \mathrm{s}$. Demographic data were recorded, including patient age, sex, previous major conditions, and the continence of the aortic valve. This study was approved by the internal review board of Nanjing Drum Tower Hospital. Written informed consent was obtained from all the participants.

All CT scans with DICOM data were uploaded to a Picture Archiving And Communication System (PACS) workstation and reconstructed using an EndoSize Workstation (Therenva, Nanjing, France). A centerline of flow (CLF) was generated using the well-proven centerline algorithms. The calculated centerline was confirmed manually by scrolling through the images in the sagittal, axial, and coronal planes, ensuring that it accurately reflected the center point of the arterial lumen. A two-dimensional format was reconstructed perpendicular to the CLF. ${ }^{15}$ Important anatomic landmarks, including primary ET and STJ, were identified. ET was accurately determined by the evidence of a clear region of intimal disruption, and contrast existed above or below the tears within the aortic wall (Figure 1). The aorta was divided into the greater curvature, lesser curvature, and anterior and posterior segments, and the location of the ET was classified

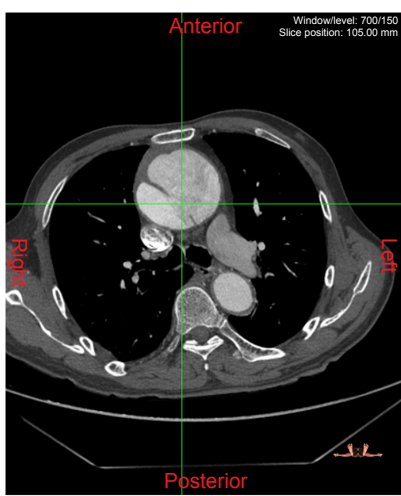

Axial plane

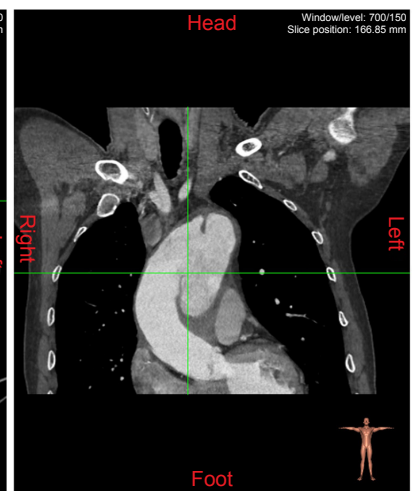

Coronal plane

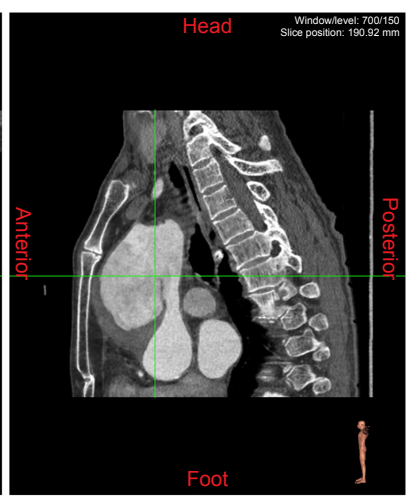

Sagittal plane

Figure I The origin of the axis indicates defined location of intimal tear.

Note: The presence of a region of intimal disruption can be viewed in three planes (axial, coronal, and sagittal planes) in the same place. 

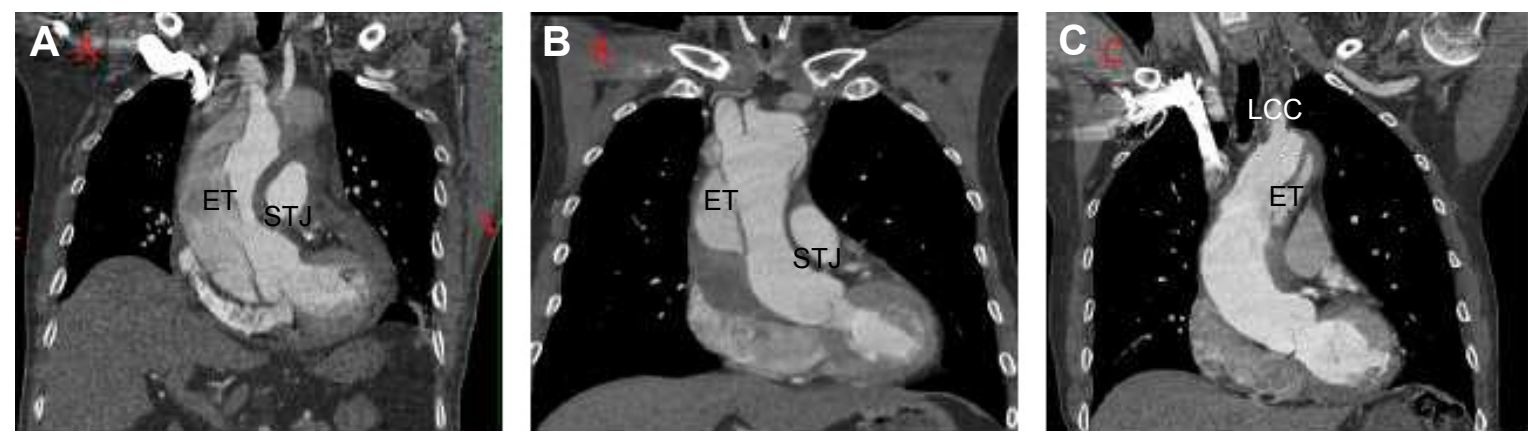

Figure 2 Various locations of the ETs.

Notes: (A) ET was close to STJ and did not offer a suitable proximal landing zone for the endograft. (B) ET located in the middle of the ascending aorta was an ideal case for endovascular treatment. (C) ET was located close to LCC, which led to the absence of suitable distal landing zone, a branched endograft or hybrid, or chimney procedure is necessary to maintain perfusion to the branch vessels.

Abbreviations: ET, entry tear; LCC, left common carotid artery; STJ, sinotubular junction.

into one of these four areas. ${ }^{8}$ The STJ was defined as the place where the sinus of Valsalva became the tubular ascending aorta. STJ was selected as the zero point for the aorta, and ET location was defined in terms of distance from proximal ET to the zero point (Figure 2).

Two experienced reviewers performed aortic measurements. The proximal native vasculature reference point was STJ. The distances between STJ and ET, STJ and the closest coronary artery, STJ and the tip of aortic valve, STJ and IA, and ET and IA, as well as the length of ET were measured. The distance from STJ to the right femoral artery was also recorded. Diameter measurements were obtained from intima to intima (inner wall to inner wall) for a circular aortic true lumen. If the perpendicular cross-sectional shape of the true lumen was elliptical or crescentic rather than circular, additional mathematical modeling (maximum + minimum diameter) $/ 2$ was required to obtain correct diameter (Figure 3). Then, the diameters at STJ level, ET level, proximal to IA, proximal to left common carotid artery, and proximal to left subclavian artery were obtained from the reconstructions perpendicular to the CLF. Descending thoracic aorta maximal diameter, abdominal aorta maximal diameter, and external iliac minimal diameter were measured. Aortic branch vessel malperfusion was also noted.

The potential candidacy for endovascular therapy was defined in our previous report: proximal landing length (distance between STJ and ET) $\geq 20 \mathrm{~mm}$, distal landing zone length (distance between ET and IA) $\geq 20 \mathrm{~mm}$, presence of available access iliofemoral vessel (diameter $>7 \mathrm{~mm}$ ), absence of severe aortic regurgitation (grade 3 or 4), and coronary bypass originating from the ascending aorta. ${ }^{10}$ Anatomical data of the suitable patients were used to determine the endograft configurations for ascending aorta. Proximal diameter and distal diameter of the endograft were selected based on the diameter measured at ET and IA levels, with $10 \%$ oversizing with respect to the true lumen, but not exceeding the original aortic diameter. The maximum length of the endograft was the distance from STJ to IA, in order not to cover the supra-aortic trunk or potentially damage normal structure of aortic root. Considering the difficulty in accurate deployment in the ascending aorta, in our opinion, the length of the endograft might be slightly shorter than the distance from STJ to IA but should not be less than the sum of the minimum landing zone and the length of ET.

Statistical analysis was performed using SAS software (Version 9.1; SAS Institute Inc., Cary, NC). Quantitative data were represented as the mean \pm standard deviation, whereas categorical and ordinal data were reported as frequencies and percentages. Differences between the mean values of the group were assessed by Student's $t$-test. The suitable rates of different pathologies were compared using chi-square analysis. $P$-values $<0.05$ were considered statistically significant. The analysis of measurement method comparison data was performed to compare intraobserver variability according to Bland and Altman. ${ }^{16}$

\section{Results}

From January 2008 to June 2014, 182 patients diagnosed with TAAD had visited our hospital. Of them, 56 patients were excluded from the study due to suboptimal CT scans and retrograde TAAD. The remaining 126 patients were adequate to allow assessment of anatomic characteristics, with a mean age of $52.1 \pm 7.6$ years. Of these patients, 89 patients $(70.6 \%)$ were male, and $103(81.7 \%)$ and $39(30.9 \%)$ patients had hypertension and diabetes, respectively. A history of smoking was found in $77(61.1 \%)$ patients.

Two reviewers' measurements were compared to assess variability, and there were only minor differences in the measured data due to the standardized CLF techniques used, and an excellent interobserver repeatability coefficient was 

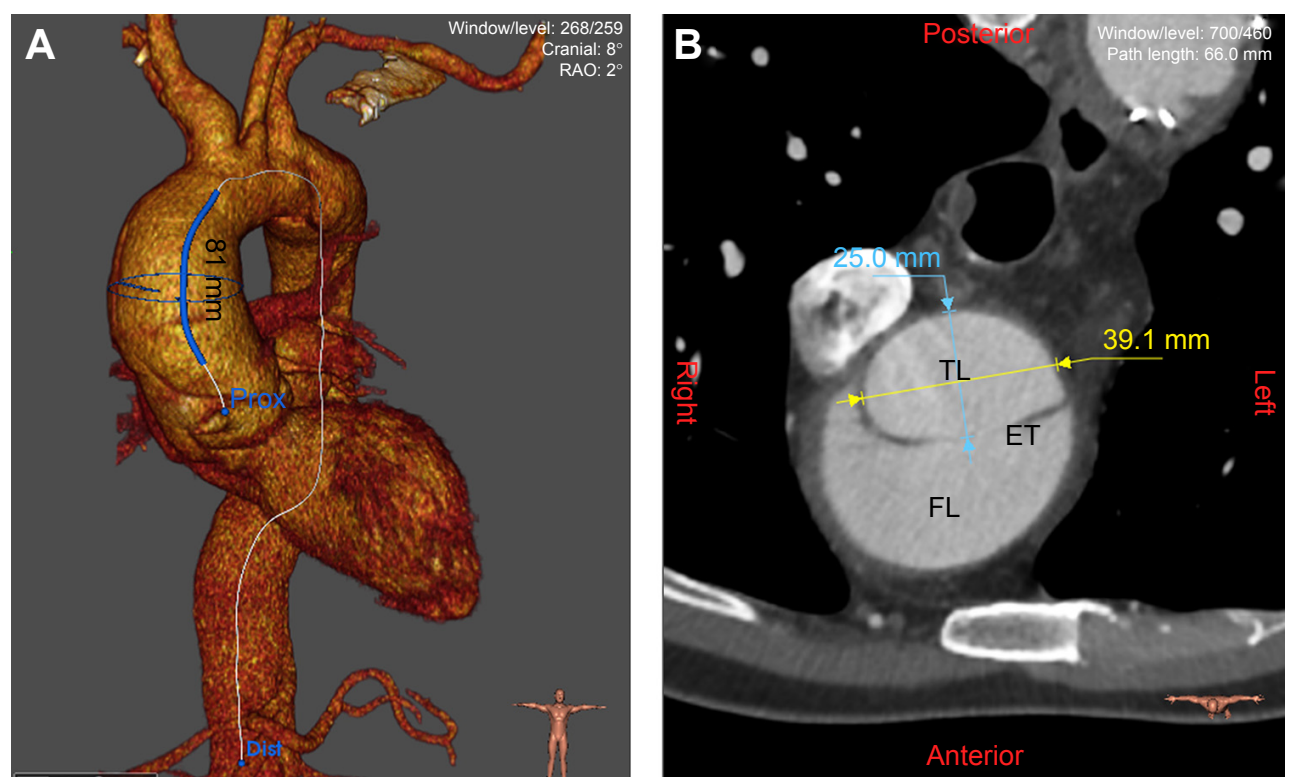

Figure 3 Aortic measurements.

Notes: (A) A successful generation of a CLF and the distance from STJ to IA was $8 \mathrm{I} \mathrm{mm}$. (B) A plane perpendicular to the CLF where the circle locates in (A) at the ET level. If the cross-sectional shape of the true lumen at ET level was elliptical or even crescentic rather than circular, mathematical modeling was done, (maximum + minimum diameter)/2, to obtain correct diameter.

Abbreviations: CLF, centerline of flow; ET, entry tear; FL, false lumen; IA, innominate artery; STJ, sinotubular junction; TL, true lumen.

obtained (Kappa 0.79), indicating no significant differences between the observers $(P>0.05)$.

ETs were located $19.1 \pm 22.6 \mathrm{~mm}$ distal to the STJ and $38.2 \pm 25.3 \mathrm{~mm}$ proximal to IA, and the detailed distribution of ETs is clearly indicated in Figure 4. It was also found that most ETs were commonly located along the greater curvature of the ascending aorta ( 88 patients, $69.8 \%$ ), with the minority occurring along the lesser curvature (15 patients, $11.9 \%$ ), the posterior aorta (13 patients, $10.3 \%$ ), and the anterior segments (ten patients, $8.0 \%$ ). All morphological

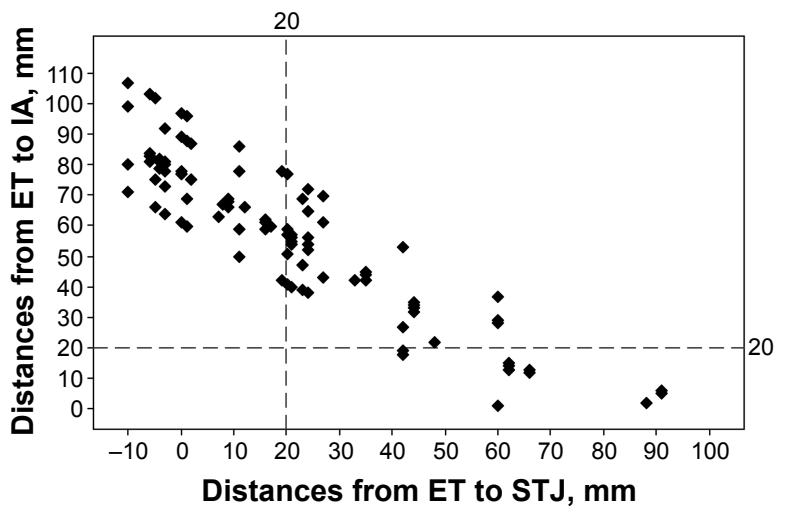

Figure $4 \mathrm{~A}$ scatter plot showing the locations of the ET.

Notes: The distance of ET is represented in millimeters relative to the STJ ( $x$ axis) and IA ( $y$ axis). The dashed lines at $20 \mathrm{~mm}$ distal to STJ and proximal to IA represent the minimum distances required for proximal and distal landing zone based on our selection criterion, so the upper right quadrant represents the potential candidacy for endovascular treatment. Patients in the lower right and upper left quadrants have unfavorable anatomy due to insufficient distal and proximal landing zones.

Abbreviations: ET, entry tear; IA, innominate artery; ST], sinotubular junction. and anatomical criteria results are summarized in Table 1. When tears were located distally to STJ, the average distance from the STJ to the highest coronary artery orifice was $8.9 \pm 2.3 \mathrm{~mm}$ (range, $5.0-13.8 \mathrm{~mm}$ ), and that from the STJ to the aortic valve in the center of the aorta was $17.4 \pm 4.1 \mathrm{~mm}$ (range, 10.2-29.3 mm).

According to the assumed criteria, 48 (38.1\%) patients were deemed to be suitable for endovascular treatment. No proximal landing zone (33 patients), no distal landing zone (12 patients), disturbed aortic valve (19 patients), coronary bypass originating from the ascending aorta (ten patients), and absence of available access vessels (four patients) were considered contraindications of an endovascular repair. These excluded patients are listed in Table 2. For these 12 patients with ET close to the origin of IA (length $<20 \mathrm{~mm}$ ), the distal landing zones could be extended via extra-anatomic carotid-carotid bypass, in situ fenestration, or using an arch-branched stent graft, then the percentage of patients suitable for endovascular repair increased up to $47.6 \%$.

Besides that, six PAU patients with ET located in the ascending aorta were included in this study. ETs of these patients were located $40.8 \pm 27.8 \mathrm{~mm}$ distal to the STJ. Among these six patients, four (66.7\%) were anatomical candidates for endovascular repair, and the rest two were unsuitable patients due to absence of the distal landing zone or coronary bypass originating from the ascending aorta, 
Table I Morphological and anatomical measurements results

\begin{tabular}{|c|c|c|c|}
\hline & $\begin{array}{l}\text { Mean } \\
(\mathrm{mm})\end{array}$ & $\begin{array}{l}\text { SD } \\
(\mathrm{mm})\end{array}$ & $\begin{array}{l}\text { Range } \\
(\mathrm{mm})\end{array}$ \\
\hline \multicolumn{4}{|l|}{ Aorta at STJ level } \\
\hline True lumen & 35.4 & 2.7 & $30.4-40.7$ \\
\hline Total aortic diameter & 35.8 & 2.6 & $31.4-40.8$ \\
\hline \multicolumn{4}{|l|}{ Aorta at the proximal ET level } \\
\hline True lumen & 37.1 & 6.6 & $26.0-46.0$ \\
\hline Total aortic diameter & 46.8 & 11.2 & $28.1-73.1$ \\
\hline \multicolumn{4}{|l|}{ Aorta at IA level } \\
\hline True lumen & 33.2 & 3.8 & $25.8-39.4$ \\
\hline Total aortic diameter & 44.0 & 7.2 & $30.1-52.7$ \\
\hline \multicolumn{4}{|l|}{ Aorta at LCC level } \\
\hline True lumen & 32.9 & 2.6 & $28.7-36.8$ \\
\hline Total aortic diameter & 37.6 & 4.1 & $32.2-44.8$ \\
\hline \multicolumn{4}{|l|}{ Aorta at LSA level } \\
\hline True lumen & 31.5 & 2.2 & $29.6-35.7$ \\
\hline Total aortic diameter & 35.8 & 2.4 & $32.2-38.8$ \\
\hline \multicolumn{4}{|c|}{ Descending thoracic aorta maximal diameter } \\
\hline True lumen & 26.3 & 6.1 & $12.7-39.1$ \\
\hline Total aortic diameter & 32.7 & 5.1 & $24.0-46.0$ \\
\hline \multicolumn{4}{|l|}{ Abdominal aorta maximal diameter } \\
\hline True lumen & 17.9 & 3.8 & $11.9-26$ \\
\hline Total aortic diameter & 23.0 & 5.8 & $15-37$ \\
\hline Left external iliac minimal diameter & 8.3 & 0.9 & $7.0-12.4$ \\
\hline Right external iliac minimal diameter & 8.4 & I.I & $7.1-12.2$ \\
\hline $\begin{array}{l}\text { Distance from the closest } \\
\text { coronary artery to STJ }\end{array}$ & 8.8 & 2.3 & $5.0-13.1$ \\
\hline $\begin{array}{l}\text { Distance from the aortic valve } \\
\text { to STJ }\end{array}$ & 17.4 & 4.1 & $12.8-23.2$ \\
\hline Distance from STJ to ET & 19.1 & 22.6 & $-|0.1-9| .5$ \\
\hline Distance from STJ to IA & 69.4 & 10.7 & $51.2-92.1$ \\
\hline Length of ET & 12.1 & 4.2 & $6.0-21.3$ \\
\hline Distance from ET to IA & 38.2 & 25.3 & $0-87.8$ \\
\hline $\begin{array}{l}\text { Distance from ST] to the right } \\
\text { femoral artery }\end{array}$ & 726.1 & 70.2 & $631.1-829.7$ \\
\hline
\end{tabular}

Abbreviations: ET, entry tear; IA, innominate artery; LCC, left common carotid artery; LSA, left subclavian artery; STJ, sinotubular junction.

respectively. However, the results were not significantly different between type A PAU and TAAD due to the small sample size ( $66.7 \%$ versus $37.5 \%, P=0.159)$.

For 126 TAAD patients, a downward trend of the true lumen diameters from the STJ level to the IA level is observed in Figure 5, indicating that conical endograft

Table 2 Details of excluded patients

\begin{tabular}{ll}
\hline Exclusion criteria & $\begin{array}{l}\text { Number of } \\
\text { the patients }\end{array}$ \\
\hline No proximal landing zone & 33 \\
No distal landing zone & 12 \\
Disturbed aortic valve & 19 \\
Coronary bypass originating from ascending aorta & 10 \\
Absence of available access vessels & 4 \\
\hline
\end{tabular}

Notes: The length of proximal landing zone $\geq 20 \mathrm{~mm}$. The length of distal sealing zone $\geq 20 \mathrm{~mm}$. The internal diameter of iliofemoral vessel $>7 \mathrm{~mm}$. Disturbed aortic valve: severe aortic regurgitation (grade 3 or 4 ).

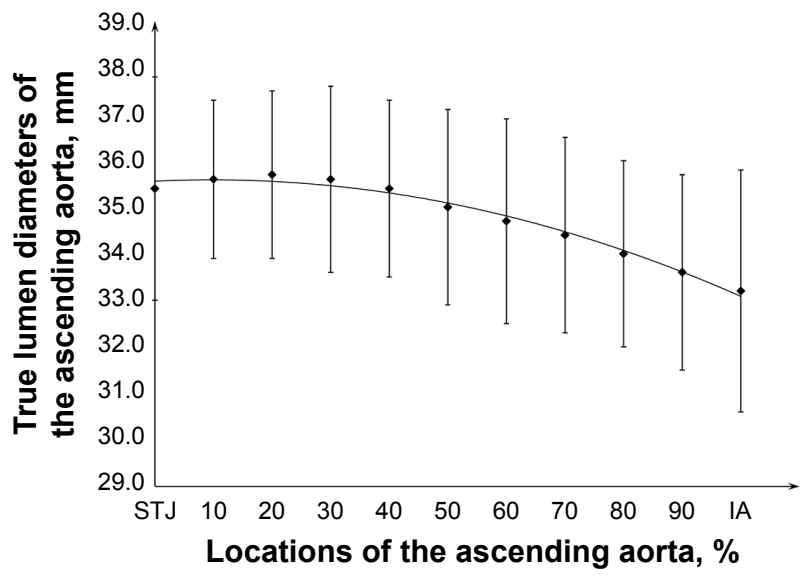

Figure $5 \mathrm{~A}$ trend graph showing the true lumen diameter versus the location of the ascending aorta.

Notes: The ascending aorta is divided into tenths from ST] to IA ( $x$ axis), and $y$ axis represents the true lumen diameters $(\mathrm{mm})$ at each level. A downward trend of the diameters is observed, which indicates that conical endografts might have advantages in the ascending aortic pathology.

Abbreviations: IA, innominate artery; ST], sinotubular junction.

might have advantages in the ascending pathology. Taking into consideration 48 patients eligible for endovascular treatment, endografts with a proximal diameter of 28,30 , $32,34,36,38,40,42,44,46,48$, and $50 \mathrm{~mm}$ were required in one $(2.1 \%)$, two $(4.2 \%)$, two $(4.2 \%)$, three $(6.2 \%)$, four (8.3\%), seven (14.6\%), nine (18.7\%), seven (14.6\%), six (12.5\%), three (6.2\%), two (4.2\%), and two (4.2\%) patients, respectively. Moreover, endografts with a distal diameter of $28,30,32,34,36,38,40,42,44$, and $46 \mathrm{~mm}$ were required in one $(2.1 \%)$, two $(4.2 \%)$, seven $(14.6 \%)$, nine $(18.7 \%)$, $12(25.0 \%)$, seven $(14.6 \%)$, four $(8.3 \%)$, three $(6.2 \%)$, two (4.2\%), and one (2.1\%) patients, respectively. Proximal diameters differed significantly from distal diameters of the endografts (39.9 versus $36.2 \mathrm{~mm}, P<0.01$ ), which also implied that conical endograft might be compatible with the ascending pathology. The lengths of the endograft required in the ascending aorta were $50,60,70,80$, and $90 \mathrm{~mm}$ in five (10.4\%), 22 (45.9\%), 13 (27.1\%), six (12.5\%), and two (4.2\%) patients, respectively.

\section{Discussion}

Successful endovascular treatment for the TAAD with ET in ascending aorta is related to the selection of suitable patients with appropriate anatomy and the interest in the production of device for such applications. The information needed for such an analysis is typically obtainable from high-resolution $\mathrm{CT}$ in conjunction with CLF analysis. ${ }^{17,18}$ Sobocinski et al ${ }^{9}$ confirmed the feasibility of endograft placement in a subset of type A dissections based on a CT study. Ronchey et $\mathrm{al}^{6}$ took advantage of high-resolution CT to tailor an aortic stent 
graft for four patients with TAAD, and their good outcomes demonstrated that a preoperative CT study was efficient and adequate to characterize this disease. Moon et $\mathrm{al}^{8}$ used an electrocardiogram-gated CT scan reconstructed at $55 \%$ or $70 \%$ of RR interval to minimize motion artifact in the ascending aorta dissection, and this four-dimensional CT with temporal resolution rendered their study more accurate. Unfortunately, electrocardiogram-gated CT was not typically performed in our emergency setting; however, this method would get more accurate anatomical characteristics and should be applied routinely in future endovascular treatment for TAAD. ${ }^{8}$ In this study, the ET location, proximal and distal landing zones, true and false lumen, patency of the false lumen, and the branch vessels as well as the extent of the dissection were evaluated with high-resolution CT and CLF analyses based on EndoSize Workstation. Our intimal tears were located 19.1 $\pm 22.6 \mathrm{~mm}$ distal to the STJ, and most of the intimal tears were located on the greater curvature. The mean length of the ascending aorta was $69.4 \mathrm{~mm}$, which is slightly shorter but without significant difference on comparison of the mean length of $70.5 \mathrm{~mm}$ reported in another study. ${ }^{8}$ Thus, there was not much difference in morphological characteristics between the Chinese population and other races, ${ }^{8,9}$ indicating that future devices could be designed as a whole.

The anatomical characteristics were then assessed to determine the suitability of endovascular treatment for such situations according to our criteria. The results showed that, in 126 TAADs with primary ET in ascending aorta, $48(38.1 \%)$ patients were anatomically suitable for endovascular treatment. Previous studies have proven that endovascular suitability was dependent mainly on an appropriate landing zone and good aortic valve function. ${ }^{6-10}$ As no fenestrated or branched endografts were commercially available to allow flow into coronary arteries, we considered that suitable length of the proximal landing zone were essential, and $20 \mathrm{~mm}$ as the minimum proximal length was enough for endograft to seal ET. Patients with inadequate proximal landing zones lead to a considerable risk of obstruction of the coronary, hemodynamic forces in this arterial segment, and the potential for fatal retrograde dissection. ${ }^{3,5}$ Patients with severe aortic regurgitation (grade 3 or 4 ) were considered unfit for endovascular repair. Although transcatheter aortic valve implantation has become a feasible therapeutic option for the management of severe aortic regurgitation, ${ }^{19}$ no study has combined this technology with endograft to treat TAAD associated with severe aortic regurgitation. So grade 3 or 4 aortic regurgitation was considered a contraindication for endovascular treatment.
In the study, we thought four (12.7\%) patients were difficult and at high risk for endovascular repair because of the absence of adequate femoral vessels (24 French). Patients without adequate femoral vessel could use the left carotid artery as an access, ${ }^{20}$ alternatively, a small thoracotomy could be used to deploy the endograft through the apex of the heart. ${ }^{21}$ For those 12 patients with ET located near the innominate trunk and absence of distal sealing zone (length $<20 \mathrm{~mm}$ ), a branched endograft, hybrid, or telescope technique was efficient to maintain perfusion to the branch vessels while covering ET. ${ }^{22}$ Finally, the percentage of patients suitable for thoracic endovascular aortic repair would increase up to $50.1 \%(64 / 126)$. However, the risk of performing endovascular repair would increase if access to femoral artery and distal landing zone was not available. ${ }^{9}$

It is worth mentioning that PAU has been acknowledged as a pathologic variant of false-lumen aortic dissection, and six type A PAU patients (4.8\%) with ET located in the ascending aorta were included in this study. Of these six patients, four $(66.7 \%)$ were anatomical candidates for endovascular repair. The higher suitable rate in PAU patients indicated that endovascular repair might have advantages in this pathology, but with a small sample size, the result was not statistically significant on comparison of TAAD patients.

Endograft placement in the ascending aorta may be difficult because the proximal and distal landing zones are very close to STJ and IA. No commercially available endovascular treatment modalities designed for TAAD increase additional challenge to the procedure. It is apparent that the ascending aortic pathologies need specific endograft solutions. ${ }^{5}$ Thus, the development of novel devices for TAAD is fundamental before these techniques can be widely adopted. In this study, we first defined the endograft configurations for the ascending aorta, which could not be replaced by off-license techniques using the aortic cuffs. ${ }^{12}$ The anatomical characteristics of TAAD patients suitable for endovascular treatment were used for the analysis of the endograft configurations. Proximal and distal diameters of the endograft were based on diameters measured at ET and IA levels with oversizing of $10 \%$. Ronchey et $\mathrm{al}^{6}$ and Nordon et $\mathrm{al}^{12}$ adopted a maximum oversizing of $10 \%$ relative to the native true lumen at the site of the proximal seal zone to minimize aortic trauma. Their experiences show that this technique is feasible in select patient categories and seems to be associated with favorable results. ${ }^{6,12}$ Significant oversizing was avoided to minimize risk of inadvertent retrograde dissection while placing the endograft in an aorta with a highly fragile intima, and; aortic 
dilatation due to excessive oversizing might be associated with aortic valve insufficiency. ${ }^{23}$ More importantly, we found that conical devices were needed in the ascending pathology, where the true lumen at the IA level was usually smaller than that at STJ and ET levels. The average length of endograft for our TAAD was $67.2 \pm 11.1 \mathrm{~mm}$, which was shorter than the available devices for the descending aorta. When tears were located distal to STJ, the average distance from the STJ to the highest coronary artery orifice was $8.9 \pm 2.3 \mathrm{~mm}$, and the average distance from the STJ to the aortic valve in the center of the aorta was $17.4 \pm 4.1 \mathrm{~mm}$. These measurements had implications for proximal device design, meaning the bare springs frequently used in descending aortic endograft must be questioned given that the potential to compromise aortic valve closure coupled with the likely failure of apposition of such stents to the wall of nontubular aortic root. ${ }^{11}$ Therefore, in our opinion, shorter, larger, and bare spring-free conical endograft was preferred to seal the ET, while presenting no harm to the fundamentally weak aorta. In some cases, a mismatch may exist between the length of the inner and outer curvature of the ascending aorta, which might easily result in a kink of the graft. Instead of using one graft, two- or threestacked grafts $5 \mathrm{~cm}$ long could solve this problem. ${ }^{11}$

Developments of the delivery system and mechanism of deployment compatible with ascending pathology were fundamental. The delivery system should have a long shaft due to the distance from the femoral artery $(726.1 \pm 70.2 \mathrm{~mm})$ and needs to be flexible to negotiate the arch and maintain torque control. The tip of the device must cross the aortic valve and sit in the left ventricle at deployment, and short tip could limit cardiac trauma. ${ }^{20}$ Easier-use deployment mechanism allows more guided placement and reduces "windsock" effect. The endograft for the ascending aorta might be semideployed at first. A constraining loop around the body of the graft enables repositioning of the stent graft, and then the constraining wire and loop are removed.

To permit exact graft deployment and prevent accidental covering of the coronary arteries, the temporary rapid ventricular pacing (heart rate 180 beats/min) was used to decrease blood pressure and cardiac output. This technique has been described and used by many authors as a means of ensuring accurate deployment of the endograft. ${ }^{6}$ While some advocated partial right atrial inflow occlusion for controlled systemic hypotension during thoracic endovascular aortic repair. ${ }^{24}$ Interestingly, Lu et $\mathrm{al}^{7}$ found that delivery system nose cone contact with the left ventricular wall induced ventricular tachycardia or fibrillation, resulting in hypotension and decreased cardiac output that allowed the endograft to be deployed accurately. The use of intraoperative transesophageal echography has been recommended to assist with precise endograft deployment, especially when the proximal deployment location is very close to the coronary sinus. ${ }^{25}$

Limitations of this study should be acknowledged. Small adequate patients were included for analysis of the endograft configurations. Diameters of the endograft for TAAD were based on the measurements on gated CT scans, because it has been shown that diameters of the thoracic aorta can change by up to $17.8 \%$ through the cardiac cycle.$^{26}$ Endografts designed for TAAD should consider the mobile diameters of the pulsating ascending aorta. Above all, this study was just a purely theoretical study exploring the endograft configurations for TAAD, and future studies are mandatory.

\section{Conclusion}

Endovascular development offered a therapeutic modality for cases of surgically untreatable TAAD, especially in anatomically suitable patients and the older patients. We suggested that shorter, larger, and bare spring-free conical endografts were preferred in the ascending aortic pathology. Developments of the delivery system and mechanism of deployment compatible with ascending pathology were also fundamental. However, this study was just a purely theoretical study exploring the endograft configurations for TAAD, and further studies were mandatory.

\section{Acknowledgment}

This work was supported by Nanjing Medical Science and Technique Development Foundation (QRX11188 and JQX14002).

\section{Disclosure}

The authors report no conflicts of interest in this work.

\section{References}

1. Trimarchi S, Eagle KA, Nienaber CA, et al; International Registry of Acute Aortic Dissection Investigators. Role of age in acute type A aortic dissection outcome: report from the International Registry of Acute Aortic Dissection (IRAD). J Thorac Cardiovasc Surg. 2010;140(4):784-789.

2. Tsai TT, Evangelista A, Nienaber CA, et al; International Registry of Acute Aortic Dissection (IRAD). Long-term survival in patients presenting with type A acute aortic dissection: insights from the International Registry of Acute Aortic Dissection (IRAD). Circulation. 2006;114(1 suppl): 1350-1356.

3. Senay S, Alhan C, Toraman F, Karabulut H, Dagdelen S, Cagil H. Endovascular stent-graft treatment of type A dissection: case report and review of literature. Eur J Vasc Endovasc Surg. 2007;34(4):457-460.

4. Ye C, Chang G, Li S, Hu Z, Yao C, Wang S. Endovascular stent-graft treatment for Stanford type A aortic dissection. Eur J Vasc Endovasc Surg. 2011;42(6):787-794. 
5. Metcalfe MJ, Karthikesalingam A, Black SA, Loftus IM, Morgan R, Thompson MM. The first endovascular repair of an acute type A dissection using an endograft designed for the ascending aorta. $J$ Vasc Surg. 2012;55(1):220-222.

6. Ronchey S, Serrao E, Alberti V, et al. Endovascular stenting of the ascending aorta for type A aortic dissections in patients at high risk for open surgery. Eur J Vasc Endovasc Surg. 2013;45(5):475-480.

7. Lu Q, Feng J, Zhou J, et al. Endovascular repair of ascending aortic dissection: a novel treatment option for patients judged unfit for direct surgical repair. J Am Coll Cardiol. 2013;61(18):1917-1924.

8. Moon MC, Greenberg RK, Morales JP, et al. Computed tomographybased anatomic characterization of proximal aortic dissection with consideration for endovascular candidacy. J Vasc Surg. 2011;53(4): 942-949.

9. Sobocinski J, O’Brien N, Maurel B, et al. Endovascular approaches to acute aortic type A dissection: a CT-based feasibility study. Eur J Vasc Endovasc Surg. 2011;42(4):442-447.

10. Huang C, Zhou M, Liu Z, et al. A computed tomography-based study exploring the feasibility of endovascular treatment of type A aortic dissection in Chinese population. J Endovasc Ther. 2014;21(5): $707-713$.

11. Kolvenbach RR, Karmeli R, Pinter LS, et al. Endovascular management of ascending aortic pathology. J Vasc Surg. 2011;53(5):1431-1437.

12. Nordon IM, Hinchliffe RJ, Morgan R, Loftus IM, Jahangiri M, Thompson MM. Progress in endovascular management of type A dissection. Eur J Vasc Endovasc Surg. 2012;44(4):406-410.

13. Preventza O, Henry MJ, Cheong BY, Coselli JS. Endovascular repair of the ascending aorta: when and how to implement the current technology. Ann Thorac Surg. 2014;97(5):1555-1560.

14. Rylski B, Blanke $\mathrm{P}$, Beyersdorf $\mathrm{F}$, et al. How does the ascending aorta geometry change when it dissects? J Am Coll Cardiol. 2014;63(13): 1311-1319.

15. O'Neill S, Greenberg RK, Resch T, et al. An evaluation of centerline of flow measurement techniques to assess migration after thoracic endovascular aneurysm repair. J Vasc Surg. 2006;43(6):1103-1110.

16. Bland JM, Altman DG. Statistical methods for assessing agreement between two methods of clinical measurement. Lancet. 1986;1(8476): $307-310$.
17. Kaladji A, Lucas A, Kervio G, Haigron P, Cardon A. Sizing for endovascular aneurysm repair: clinical evaluation of a new automated three-dimensional software. Ann Vasc Surg. 2010;24(7):912-920.

18. Rajani RR, Johnson LS, Brewer BL, et al. Anatomic characteristics of aortic transection: centerline analysis to facilitate graft selection. Ann Vasc Surg. 2014;28(2):433-436.

19. Pesarini G, Bedogni F, Ribichini F. "Valve in valve" implantation of two self-expandable transcatheter aortic valves in a patient with aortic root aneurysm and massive aortic regurgitation: "a new TAVI option". Catheter Cardiovasc Interv. 2014;83(5):836-840.

20. Lin PH, Kougias P, Huynh TT, Huh J, Coselli JS. Endovascular repair of ascending aortic pseudoaneurysm: technical considerations of a common carotid artery approach using the Zenith aortic cuff endograft. J Endovasc Ther. 2007;14(6):794-798.

21. Kappert U, Ghazy T, Ouda A, Hoffmann RT, Simonis G, Matschke K. Transapical endovascular stenting of penetrating atherosclerotic ulcer of ascending aorta. Ann Thorac Surg. 2013;96(4):e101-e103.

22. Bergeron P, Mangialardi N, Costa P, et al. Great vessel management for endovascular exclusion of aortic arch aneurysms and dissections. Eur J Vasc Endovasc Surg. 2006;32(1):38-45.

23. Zhang H, Li M, Jin W, Wang Z. Endoluminal and surgical treatment for the management of Stanford Type A aortic dissection. Eur J Cardiothorac Surg. 2004;26(4):857-859.

24. Lee WA, Martin TD, Gravenstein N. Partial right atrial inflow occlusion for controlled systemic hypotension during thoracic endovascular aortic repair. J Vasc Surg. 2008;48(2):494-498.

25. Ihnken K, Sze D, Dake MD, Fleischmann D, Van der Starre P, Robbins R. Successful treatment of a Stanford type A dissection by percutaneous placement of a covered stent graft in the ascending aorta. J Thorac Cardiovasc Surg. 2004;127(6):1808-1810.

26. Muhs BE, Vincken KL, van Prehn J, et al. Dynamic cine-CT angiography for the evaluation of the thoracic aorta; insight in dynamic changes with implications for thoracic endograft treatment. Eur J Vasc Endovasc Surg. 2006;32(5):532-536.
Clinical Interventions in Aging

\section{Publish your work in this journal}

Clinical Interventions in Aging is an international, peer-reviewed journal focusing on evidence-based reports on the value or lack thereof of treatments intended to prevent or delay the onset of maladaptive correlates of aging in human beings. This journal is indexed on PubMed Central, MedLine,

\section{Dovepress}

CAS, Scopus and the Elsevier Bibliographic databases. The manuscript management system is completely online and includes a very quick and fair peer-review system, which is all easy to use. Visit http://www.dovepress. com/testimonials.php to read real quotes from published authors. 